\title{
Gas-solid Erosion Wear Characteristics of Two-phase Flow Tee Pipe
}

\author{
Jin HU*, Hao ZHANG**, Jie ZHANG***, Shiwei NIU****, Wenbo CAI***** \\ *School of Mechatronic Engineering, Southwest Petroleum University, 610500 Chengdu, P. R. China, \\ E-mail: $23880441 @ q q . c o m$ \\ **School of Mechatronic Engineering, Southwest Petroleum University, 610500 Chengdu, P. R. China, \\ E-mail:3059849736@qq.com \\ ***Institute of Metal Materials Abrasion Control and Moulding, 471003 Luoyang, P. R. China, \\ E-mail: longmenshao@163.com \\ ****School of Mechatronic Engineering, Southwest Petroleum University, 610500 Chengdu, P. R. China, \\ E-mail: $565060448 @ q q . c o m$ \\ *****School of Mechatronic Engineering, Southwest Petroleum University, 610500 Chengdu, P. R. China, \\ E-mail:871003912@qq.com \\ crossref http://dx.doi.org/10.5755/j02.mech.27478
}

\section{Introduction}

Erosion action of the pipe wall surfaces is regarded as a kind of wash and corrosion [1]. When the high-speed gas with particles flows through the wall of the tee pipe, the particles hit the wall and take away part of the wall quality, causing the tee pipe erosion [2]. The general form of erosion on the wall is that erosion pits and the uneven surface. The severer tee pipe erosion is formed perforation and even cause the tee pipeline to break [3]. However, the erosion process of the tee pipe is complex and is affected by the flow velocity, particle mass flow rate, particle diameter, impact angle and the target material properties [4].

Zeng and Zhang studied the causes of rare erosion indentation, which were analysed from the trajectory and secondary flow of the particles by the CFD-DEM two-way coupling method [5]. $\mathrm{Xu}$ and Sun found the erosion of the polar seawater pipeline wall by ice particles under the condition of vibration [6]. Wang et al simulated the relationship between the secondary flow and the particle trajectory, which was analysed by establishing a numerical model and focusing on the relationship between the secondary flow and the particle trajectory [7]. Qiao and Cheng studied the corrosion failure of elbow pipeline and welds in the natural gas gathering and transportation pipeline [8]. Pei et al. worked out the numerical analysis on the maximum fluid-solid coupling erosion part in the elbow pipeline, and studied the flow field distribution, particle motion trajectory and the relationship between the maximum erosion rate and the influencing factors through computational fluid dynamics [9]. Zhou and Liu studied the influence of solid particle shape and fluid swirling intensity on the erosion at the elbow [10].

Zhang and Bai studied the failure analysis and erosion prediction on the tee joint in fracturing operation [11]. M.R. Banakermani worked out the numerical analysis and prediction of the erosion of the elbow for the gas-solid twophase flow in the elbow pipelines [12]. Akbar Arsalanloo studied the problem of particle deposition in elbow pipeline with swirling [13]. Zhu and Li studied the erosion when trapezoidal ribs are added to the outer wall of the elbow [14]. Carlos improved the design of the pipeline wall to reduce the erosion of the elbow [15]. Mazdak studied the erosion of sand particle in multiphase flow, and found a new dimensionless number for solid particle erosion. Mohammad studied the erosion of rotating solid particles in gas-solid two- phase flow on the elbow $[16,17]$. In this paper, the tee pipe erosion was investigated, the effect of inlet diameter, inlet velocity and particle mass flow rate were discussed.

\section{Mathematical model}

Mathematical models of the study include turbulence model, particle trajectory model and erosion model [18-22]. The gas with sand can be one-way coupling or twoway coupling. The one-way coupling can save a lot of calculation time, but neglects the influence of particle motion on fluid flow. The influence of particle motion on fluid flow is taken into account in the two-way coupling, so the calculation process of two-way coupling is complicated and the calculation time is longer. The gas flow can be described by Euler method, but the movement of sand in the gas is the main object of attention in the process of erosion. In addition, Lagrange method is more suitable than Euler method to describe the trajectory of discrete phase particle, and the motion of particles obeys Newton's second law. The movement of discrete phase particles in the tee pipe is complex.

The interaction between fluid and particles can be divided into two sides: The sum of the force applied by the fluid on the particles and the torque acting on the particles. The motion of the particles can be described by the following equations:

$$
m \frac{d u}{d t}=m g+\sum F_{g}+F_{D}+F_{S L}+F_{M L}
$$

In these equations, $F_{g}, F_{D}, F_{S L}, F_{M L}$ represent the sum of the buoyancy that the particles are applied in the fluid, the fluid resistance, the Saffman lift, and the Magnus lift, N. The forces that the particles are applied in the fluid are complex. Basset force and electromagnetic force are not considered in this study. Pressure gradient force and false mass force are very small and can be ignored. Where $F_{g}$ is calculated as follows:

$$
F_{g}=-\frac{\rho_{f}}{\rho} m g,
$$

where: $\rho$ represents the density of sand, $\mathrm{kg} / \mathrm{m} 3 ; F_{D}$ is calculated as follows: 


$$
F_{D}=\frac{3 \rho_{f} m C_{D}}{4 \rho D}\left|u_{f}-u\right|\left(u_{f}-u\right)
$$

In order to save calculation time, the spherical particles with the circular degree of 1 are selected in this study. Where $C_{D}$ represents the drag coefficient when the particle is spherical, is calculated as follows:

$$
C_{D}=\left\{\begin{array}{cc}
24 \operatorname{Re}^{-1}\left(1+0.15 \operatorname{Re}_{p}^{0.687}\right), & \operatorname{Re}_{p} \leq 1000 \\
0.44, & \operatorname{Re}_{p}>1000
\end{array}\right.
$$

The Reynolds number of the particle $\left(R e_{p}\right)$ can be calculated based on the relative velocity of the particle and the fluid.

$$
R e_{p}=\rho_{f}\left|u_{f}-u\right| D_{p} / \mu
$$

In the equation, $D_{p}$ represents the diameter of the particle, $\mathrm{m} ; \mu$ represents kinetic viscosity of the fluid. The fluid in this study is nature gas, whose kinetic viscosity is $1.087 \mathrm{e}-05 . F_{S L}$ is the force that the particles are applied with the sheer flow induced by velocity gradient in the flow field, is calculated as follows:

$$
F_{S L}=1.615 D \mu_{f} R e_{s}^{0.5} C_{s l}\left[\left(u_{f}-u\right) \times \omega_{f}\right],
$$

where: $\omega_{f}$ represents the vorticity of the fluid; $R e_{s}$ represents the Reynolds number for shear flow; $C_{s l}$ represents coefficient for Saffman lift.

$$
C_{s l}=\left\{\begin{array}{cc}
(1-0.331 \sqrt{\beta}) e^{-R e_{s}} / 10+0.331 \sqrt{\beta}, & R e_{s} \leq 40 \\
0.0524 \sqrt{\beta R e_{s}}, & R e_{s}>40
\end{array}\right.
$$

$F_{M L}$ represents the Magnus lift for rotating particles, is calculated as follows:

$$
\begin{aligned}
& F_{M L}=\frac{\pi}{8} D^{3} \rho_{f} \frac{R e}{R e_{R}} C_{M L}\left[\Omega \times\left(u_{f}-u\right)\right] \\
& \operatorname{Re}_{R}=\rho_{f} D^{2}|\Omega| / \mu \\
& C_{M L}=0.45+\left(\frac{R e_{R}}{R e_{p}}-0.45\right) e^{-0.05684 R e_{R}^{0.4} R e_{p}^{0.3}}
\end{aligned}
$$

After a great deal of experiments, several erosion models have been verified and applied [23, 24]. The calculation of erosion rate by FLUENT is judged by the mass of the wall reduction, which is defined as the mass reduction within the unit time unit area of the pipe wall. There are two main reasons for reducing the mass of wall material: shearing and collision. The erosion rate is defined as follows:

$$
R_{\text {erosion }}=\sum_{p=1}^{N_{\text {particle }}} \frac{m_{p} C(d) f(\alpha) v^{b}}{A_{\text {face }}}
$$

In the equation, $C(d)$ represents the particle diameter function of solid phase particle; $f(\alpha)$ represents impact angle function; $b$ represents velocity exponent function;
$A_{\text {face }}$ represents pipeline wall surface area. When the boundary condition is set, it is necessary to set the wall properties. Then $C(d)$ general taking constant $1.8 \mathrm{e}-9, b$ general taking constant 2.6. The impact angle function is seen in Table 1 . What's more, the particle rebound model is set as follows [25]:

$$
\begin{aligned}
& e_{n}=0.993-1.76 \alpha+1.56 \alpha^{2}-0.49 \alpha^{3} \\
& e_{\tau}=0.998-1.66 \alpha+2.11 \alpha^{2}-0.67 \alpha^{3}
\end{aligned}
$$

Table 1

Impact angle function

\begin{tabular}{|c|c|c|c|c|c|}
\hline Angle $^{\mathbf{}}$ & 0 & 20 & 30 & 45 & 90 \\
\hline value & 0 & 0.8 & 1 & 0.5 & 0.4 \\
\hline
\end{tabular}

\section{Model representation}

\subsection{Geometry model and boundary conditions}

As shown in Fig. 1, a tee pipe with a length ratio of $l / L=0.5$ and a diameter ratio of $d / D=1$ was first used to simulate. The tee pipe is made of carbon steel with a density of $7850 \mathrm{~kg} / \mathrm{m}^{3}$, Young's modulus of $210 \mathrm{GPa}$ and Poisson's ratio of 0.3. The pipe diameter $D$ is a constant and the pipe diameter $d$ is a variable. The inlet of tee pipe is $\mathrm{A}$, and the outlets of tee pipe are Left_B and Right_C. Five different pipe diameters $d$ are considered to simulate the influence of the hydraulic diameter.

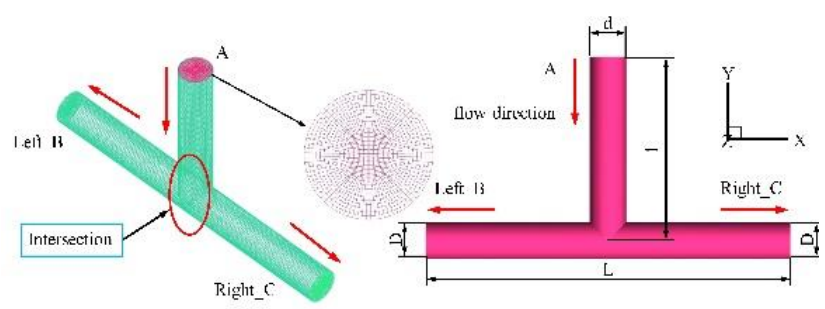

Fig. 1 Geometry and computational mesh $(d=D=60 \mathrm{~mm})$

The flow fluid is nature gas with constant density and viscosity, and the inlet velocity of gas increases to 60 $\mathrm{m} / \mathrm{s}$ with increment of $10 \mathrm{~m} / \mathrm{s}$. The inert solid medium is sand with mean diameter of $100 \mu \mathrm{m}$ and roundness of 1 and density is $1550 \mathrm{~kg} / \mathrm{m}^{3}$. The natural gas, as a continuous phase with a density of $0.6679 \mathrm{~kg} / \mathrm{m}^{3}$ and the dynamic viscosity of $1.087 \mathrm{e}-5 \mathrm{~kg} \cdot \mathrm{m}^{-1} \cdot \mathrm{s}^{-1}$. Sand is injected from the inlet pipe with particle mass flow rate ranges from $0.001 \mathrm{~kg} / \mathrm{s}$ to $0.006 \mathrm{~kg} / \mathrm{s}$ with increment of $0.001 \mathrm{~kg} / \mathrm{s}$. Thus the concentration of solid particles in gas is in the range of $3.54 \times 10^{-2}$ $2.12 \times 10^{-1}$. The DPM model is enough to obtain reasonable results in dilute flow. The inlet boundary condition is set to be velocity-inlet specified as $v=v_{0}$, while the pressure outlet describes the outlet boundary condition with a gauge pressure of $101325 \mathrm{~Pa}$. The wall shear condition is no-skip and the wall roughness is a constant of 0.5 .

\subsection{Mesh independence verification}

In order to obtain reasonable simulation results, the quality of mesh is improved by increasing the number of meshes. Fig. 2 and Fig. 3 compare respectively the flow velocity and turbulence intensity distribution of these meshes at the inlet pipe and outlet pipe. In addition, it should be 
known that these simulations used realizable k-epsilon model with the near-wall treatment of standard wall functions. In addition, the maximum erosion rate is compared with percentage change as shown in Table 2 . It is seen that the minimum percentage changes of 0 occurs between Mesh 5 and Mesh 6. However, given the question of calculating time, the number of meshes should be reduced as much as possible. Finally, Mesh 5 is a good choice with higher precision and shorter calculation time.

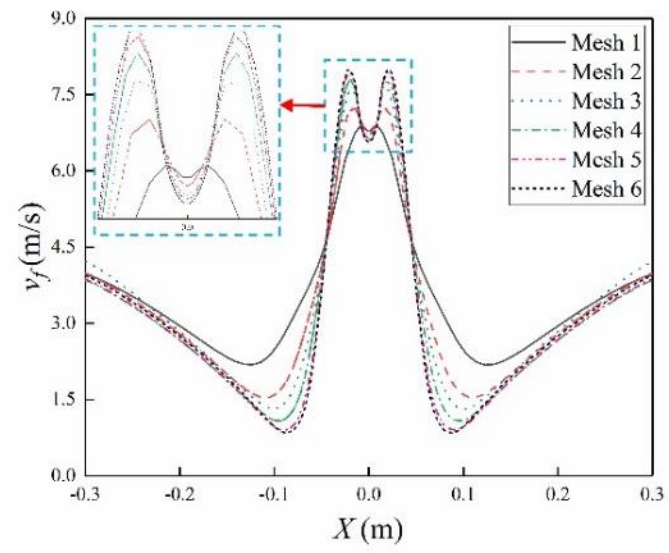

Fig. 2 Velocity at the outlet pipe $\left(d=60 \mathrm{~mm}, v_{0}=10 \mathrm{~m} / \mathrm{s}\right.$, $Q_{p}=0.001 \mathrm{~kg} / \mathrm{s}$ )

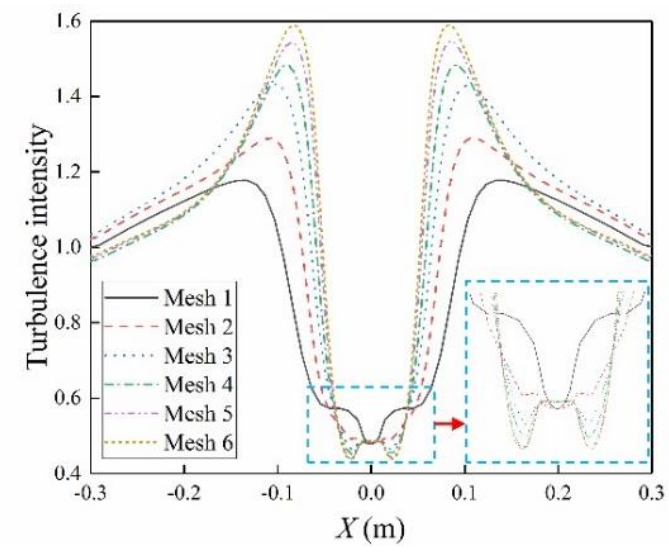

Fig. 3 Turbulence intensity at the inlet pipe $\left(d=60 \mathrm{~mm}, v_{0}\right.$ $=10 \mathrm{~m} / \mathrm{s}, Q_{p}=0.001 \mathrm{~kg} / \mathrm{s}$ )

Table 2

Mesh independence confirmations. The $e_{\max }$ of the wall surface $(d=60 \mathrm{~mm})$

\begin{tabular}{|c|c|c|c|}
\hline \multirow{2}{*}{ Mesh } & \multirow{2}{*}{ Mesh number } & \multicolumn{2}{|c|}{$e_{\max } \mathrm{kg} \cdot \mathrm{m}^{-2} \cdot \mathrm{s}^{-1}$} \\
\cline { 3 - 4 } & & Value & Percentage changes \\
\hline 1 & 26840 & $1.09 \mathrm{E}-07$ & $/$ \\
\hline 2 & 80261 & $1.15 \mathrm{E}-07$ & $5.22 \%$ \\
\hline 3 & 165744 & $1.17 \mathrm{E}-07$ & $1.71 \%$ \\
\hline 4 & 206017 & $1.22 \mathrm{E}-07$ & $4.1 \%$ \\
\hline 5 & 292688 & $1.23 \mathrm{E}-07$ & $0.81 \%$ \\
\hline 6 & 418128 & $1.23 \mathrm{E}-07$ & 0 \\
\hline
\end{tabular}

\subsection{Erosion model validation}

The comparison between calculation results and experiment results [3] by using the general erosion model is shown in Table. 3. It can be seen that the error of erosion rate of seven angles on the extrados of the elbow. Both the experimental results and the simulation results reach the maximum value at $49^{\circ}$, and the error is only $2.27 \%$. Therefore, this erosion model can be used to solve the erosion rate of the tee pipe.

Table 3

Erosion model validation. $\left(v_{0}=45.72 \mathrm{~m} / \mathrm{s}, Q_{p}=0.000208 \mathrm{~kg} / \mathrm{s}\right)$

\begin{tabular}{|c|c|c|c|}
\hline Value $^{\circ}$ & $\begin{array}{c}\text { Experiment } \\
/ \mathrm{kg} \cdot \mathrm{m}^{-2} \cdot \mathrm{s}^{-1}\end{array}$ & $\begin{array}{c}\text { Simulation } \\
/ \mathrm{kg} \cdot \mathrm{m}^{-2} \cdot \mathrm{s}^{-1}\end{array}$ & Error/\% \\
\hline 12.5 & $4 \mathrm{e}-6$ & $5.56 \mathrm{e}-6$ & 39 \\
\hline 24 & $8 \mathrm{e}-6$ & $1.27 \mathrm{e}-6$ & -84.1 \\
\hline 36 & $2.2 \mathrm{e}-5$ & $2.74 \mathrm{e}-6$ & -87.5 \\
\hline 49 & $4.4 \mathrm{e}-5$ & $4.3 \mathrm{e}-5$ & -2.27 \\
\hline 57 & $1.3 \mathrm{e}-5$ & $2.1 \mathrm{e}-5$ & 61.5 \\
\hline 68 & $3 \mathrm{e}-6$ & $7.4 \mathrm{e}-6$ & 147 \\
\hline 78 & $2 \mathrm{e}-6$ & $3.2 \mathrm{e}-6$ & 60 \\
\hline
\end{tabular}

\section{Simulation results and discussion}

\subsection{Effect of the inlet diameter}

As shown in Fig. 4, an elliptical high-pressure region is formed at the intersection of tee pipe. Furthermore, the inlet diameter corresponds to the oblong high-pressure region. The larger the inlet diameter, the larger the oblong high-pressure region. After the gas with sand enters the tee pipe and passes through the inlet pipe, it hits the bottom of the pipe intersection, and the direction of the gas changes. Thus, the instantaneous velocity of the gas is 0 , according to the Ideal Gas Law, this is a high pressure region.

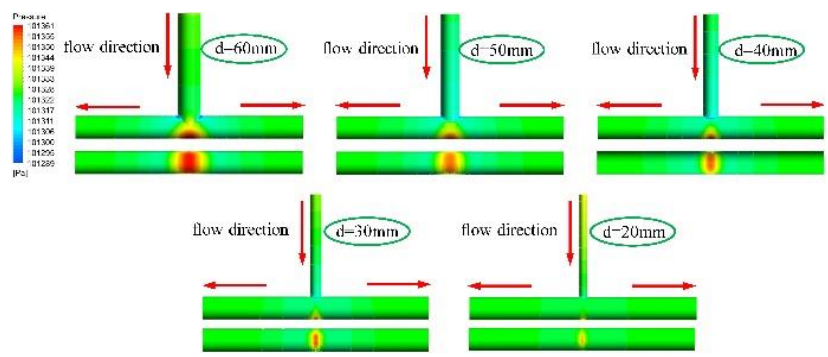

Fig. 4 Contours of pressure $\left(v_{0}=10 \mathrm{~m} / \mathrm{s}, Q_{p}=0.001 \mathrm{~kg} / \mathrm{s}\right)$

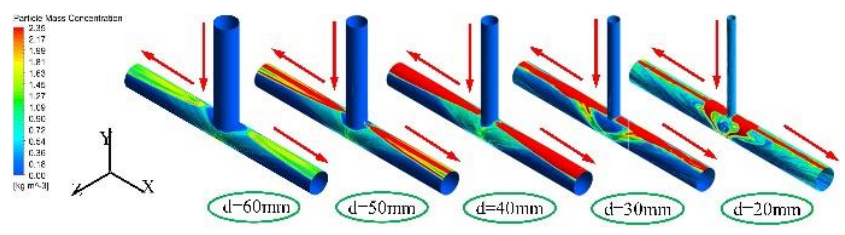

Fig. 5 Contours of particle mass concentration $\left(v_{0}=10 \mathrm{~m} / \mathrm{s}\right.$, $\left.Q_{\mathrm{p}}=0.001 \mathrm{~kg} / \mathrm{s}\right)$

Fig. 5 shows the particle mass concentration distribution at the outlet pipe of the tee tube. In dilute flow, sand moves with the fluid. Sand in the gas hits the tee pipe wall and rebound because the flow direction of the fluid in the tee pipe is limited. Different impact angles cause different rebound angles. The particle mass concentration is concentrated on the top of the outlet pipe and on both sides of the tee pipe intersection. However, the particle mass concentration distribution appears on both sides of the outlet pipe with the decrease of inlet diameter.

Fig. 6 shows the contours of erosion rate. An elliptical erosion region is formed at the bottom of the tee pipe intersection, which is the same as the position of the highpressure region. However, there is no erosion region at the outlet pipe. Sand without considering the deformation hits 
the wall, the kinetic energy of sand is changed into the potential energy of the wall. The larger the inlet diameter, the larger the region at the bottom of the tee pipe intersection directly impacted by the sand through the inlet pipe. Therefore, the elliptical erosion region becomes larger and the maximum erosion rate becomes smaller.

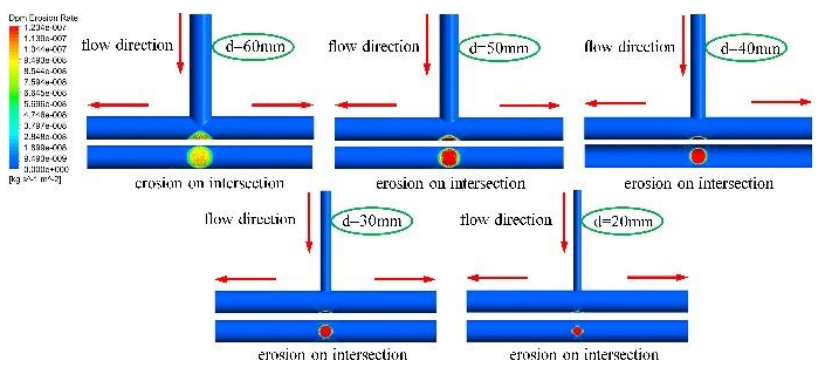

Fig. 6 Contours of erosion rate $\left(v_{0}=10 \mathrm{~m} / \mathrm{s}, Q_{\mathrm{p}}=0.001 \mathrm{~kg} / \mathrm{s}\right)$

Fig. 7 shows the relationship between maximum erosion rate and inlet diameter. The maximum erosion rate becomes smaller when the inlet diameter increases from 20 $\mathrm{mm}$ to $60 \mathrm{~mm}$ with increment of $10 \mathrm{~mm}$. As the inlet diameter increases from $20 \mathrm{~mm}$ to $40 \mathrm{~mm}$, the decrease rate of the erosion is relatively fast while it becomes slower at $d$ > $40 \mathrm{~mm}$. When the inlet diameter is $20 \mathrm{~mm}$, the maximum erosion rate is $1.29 \times 10^{-6} \mathrm{~kg} \cdot \mathrm{m}^{-2} \cdot \mathrm{s}^{-1}$. However, the maximum erosion rate is $1.23 \times 10^{-7} \mathrm{~kg} \cdot \mathrm{m}^{-2} \cdot \mathrm{s}^{-1}$ when the inlet diameter is $60 \mathrm{~mm}$. Therefore, the larger the inlet diameter, the smaller the maximum erosion rate.

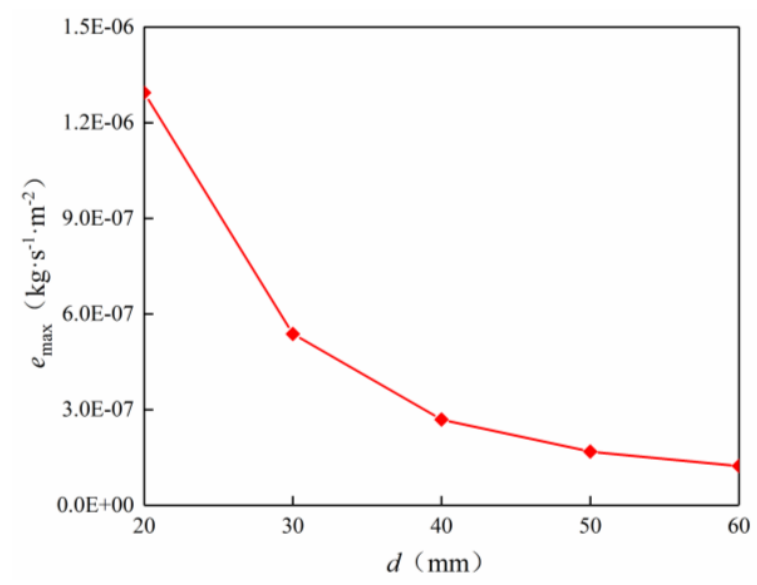

Fig. 7 Inlet diameter and the maximum erosion rate

In order to verify the particle mass concentration distribution on the sides of the outlet pipe and the effect of secondary flow vortices on the erosion area region, the turbulent intensity, velocity contours and streamlines were acquired in a normal cross-section to the tee pipe axis. Seven positions are selected as the sections, which are $X=0,30$ $\mathrm{mm}, 40 \mathrm{~mm}, 45 \mathrm{~mm}, 50 \mathrm{~mm}, 80 \mathrm{~mm}, 150 \mathrm{~mm}$ of the outlet pipe (named $l_{0}, l_{1}, l_{2}, l_{3}, l_{4}, l_{5}, l_{6}$, respectively), as shown in Fig. 8. Especially, the cases of $d=20 \mathrm{~mm}, 40 \mathrm{~mm}, 60 \mathrm{~mm}$ are chosen to examine the effect of inlet velocity.

Fig. 9 shows the contours of turbulent intensity in seven cross-sections. With the distance increases, the variation of turbulent intensity is more obvious. As shown in Fig. 9 , a, the region of maximum turbulent intensity is concentrated on the sides and bottom of the section at $l_{0}$. When the gas reaches $l_{5}$, three regions of maximum turbulent intensity are located on the top of outlet pipe. The gas continues to flow to the downstream and the regions of maximum turbulent intensity move from the top to the centre of the section. When the flow reaches $l_{6}$, three regions with the maximum turbulent intensity is formed gradually a region.

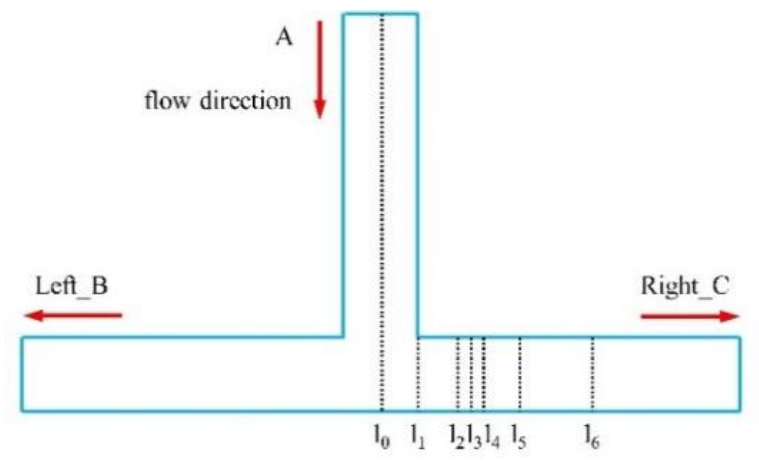

Fig. 8 Positions of seven cross-section

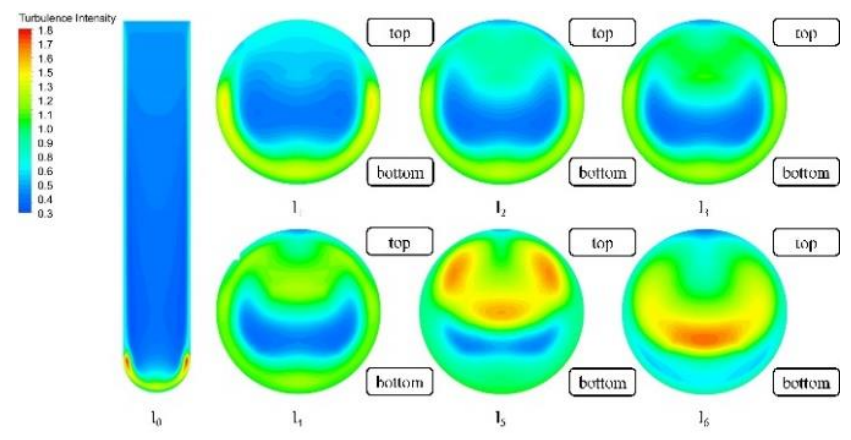

a
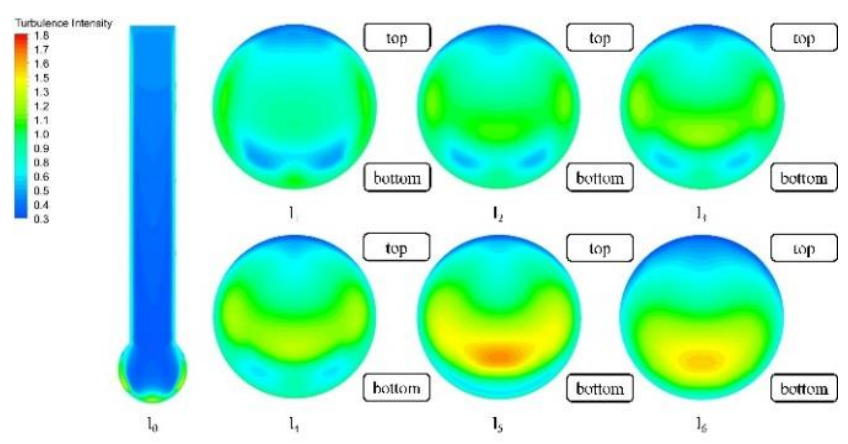

b
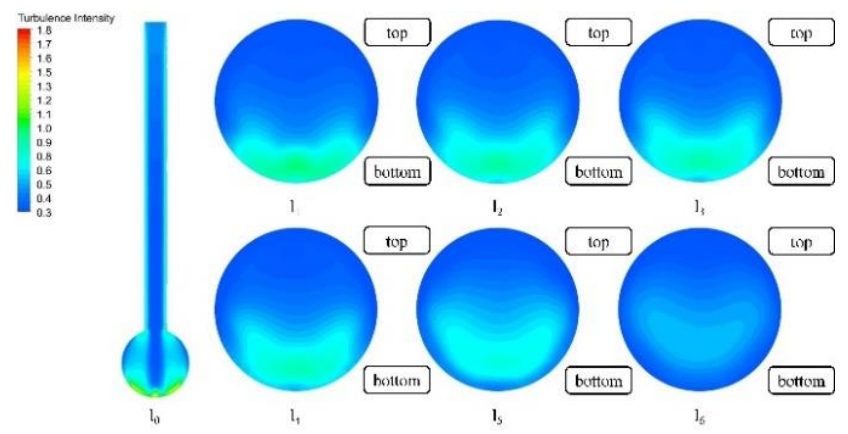

c

Fig. 9 Contours of turbulent intensity (a $-d=60 \mathrm{~mm}$; $\mathrm{b}-d=40 \mathrm{~mm} ; \mathrm{c}-d=20 \mathrm{~mm}, v_{0}=10 \mathrm{~m} / \mathrm{s}, Q_{p}=$ $=0.001 \mathrm{~kg} / \mathrm{s})$

The regions of maximum turbulent intensity are concentrated on the sides of the section at $l_{0}$ and they exist independently of each other, as shown in Fig. 9, b. When the gas reaches $l_{3}$, the region of maximum turbulent intensity is located on the centre of section. The fluid continues to flow 
to the downstream and the region of maximum turbulent intensity becomes larger. As shown in Fig. 9, c, the variation of the region of maximum turbulent intensity is small. Therefore, the larger the inlet diameter, the lower the turbulence intensity in the outlet pipe.
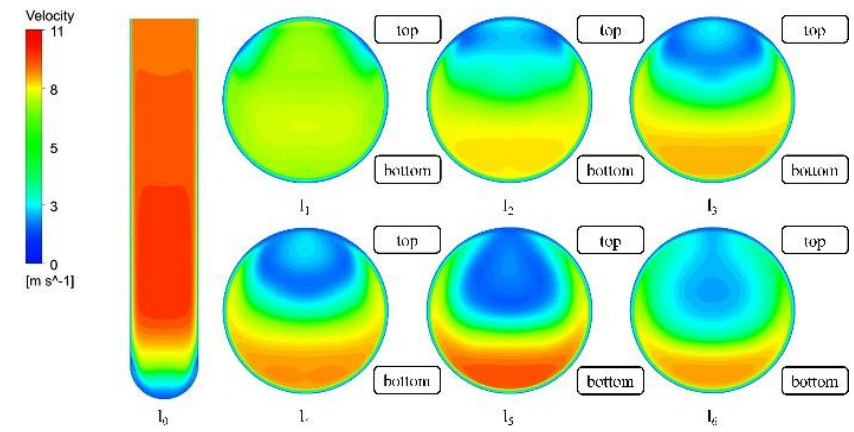

$\sum_{0}^{11}$
8
3
$\left[\mathrm{~ms}^{\mathrm{n}-1]}\right.$
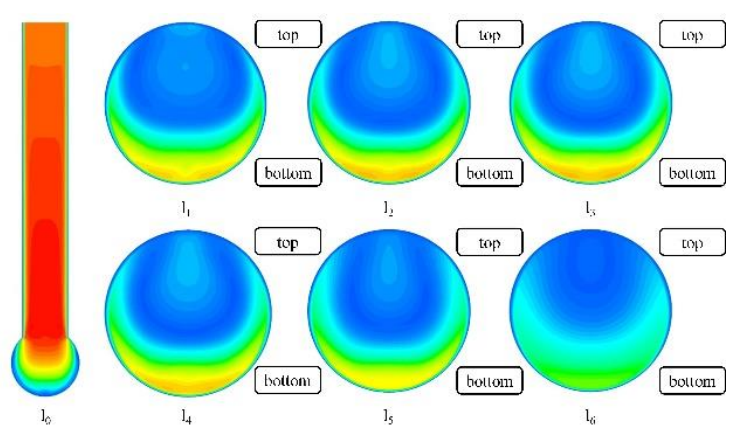

b
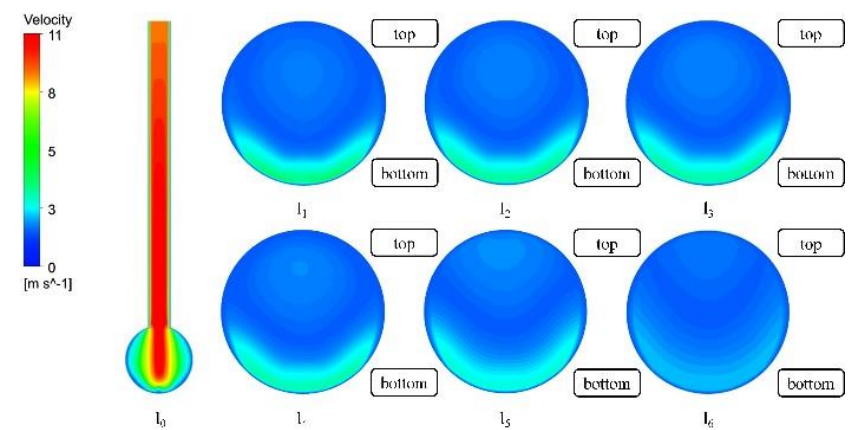

$\mathrm{c}$

Fig. 10 Contours of velocity ( $\mathrm{a}-d=60 \mathrm{~mm} ; \mathrm{b}-d=40 \mathrm{~mm}$; c - $\left.d=20 \mathrm{~mm}, v_{0}=10 \mathrm{~m} / \mathrm{s}, Q_{\mathrm{p}}=0.001 \mathrm{~kg} / \mathrm{s}\right)$

Fig. 10 shows the contours of gas velocity in seven cross-sections. The velocity gradient can be obviously observed. Initially at the section of $l_{0}$, the gas passes through the inlet pipe at high velocity and hits the bottom of the pipe intersection. Due to the different inlet diameter, the paths of gas flow in the outlet pipe are different. As shown in Fig. 10 , a, the region of high flow velocity is located on the bottom of section. When the gas reaches $l_{6}$, the gas velocity becomes the largest. However, with the gas continues to flow to downstream, the region of high flow velocity becomes small. This variation of gas velocity is regular in Fig. 10, b. Both the region of high flow velocity and the gas velocity become smaller. As shown in Fig. 10, c, the gas velocity is small and the variation of velocity is slight. When the gas reaches the outlet pipe, the smaller the inlet diameter, the slower the gas velocity in the outlet pipe.

Fig. 11 shows the streamlines of gas in seven crosssections. Because the flow direction of the gas changes at the intersection of the tee pipe, the gas flow state in the outlet pipe becomes complexity. Therefore, the streamlines of gas in seven sections were acquired to analyse the emergence and development of vortices. As shown in Fig. 11, a, there are two small vortices on the sides of outlet pipe when the gas passes through the intersection. At $l_{1}$, these two vortices become larger. At $l_{2}$ and $l_{3}$, they become larger and gradually approach the top of the section. At $l_{4}$, two vortices close to each other appear at the top of the section, while the vortices on both sides disappear. At $l_{5}$ and $l_{6}$, these two vortices become larger and gradually approach the centre of gas flow.
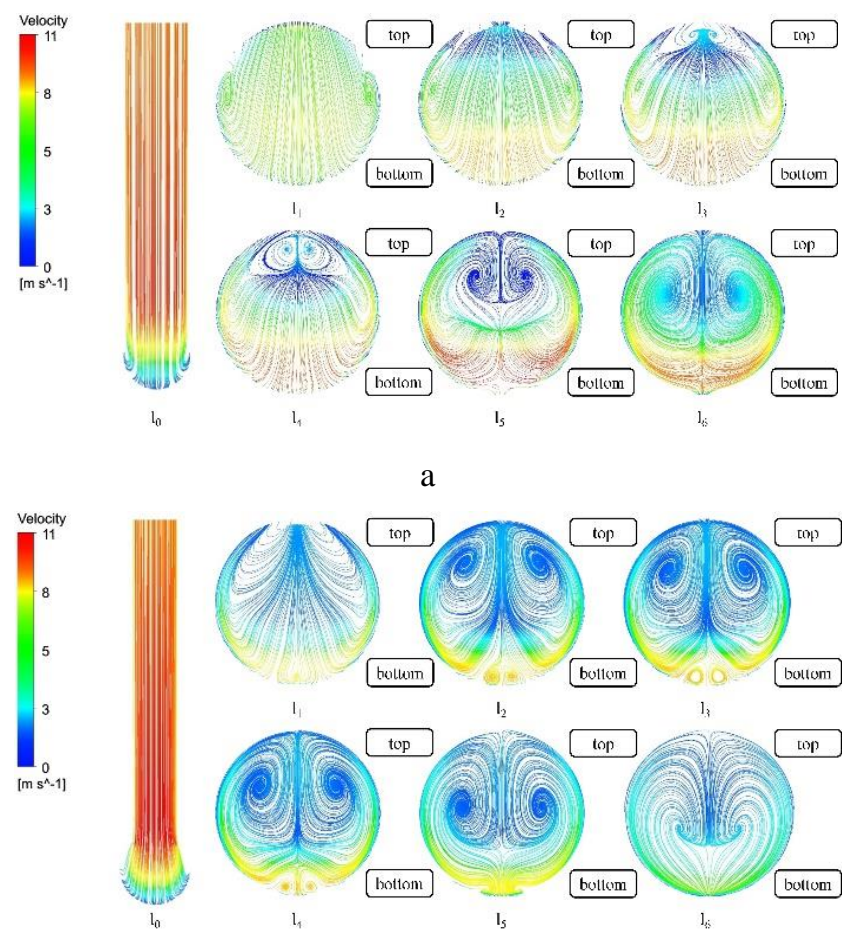

b
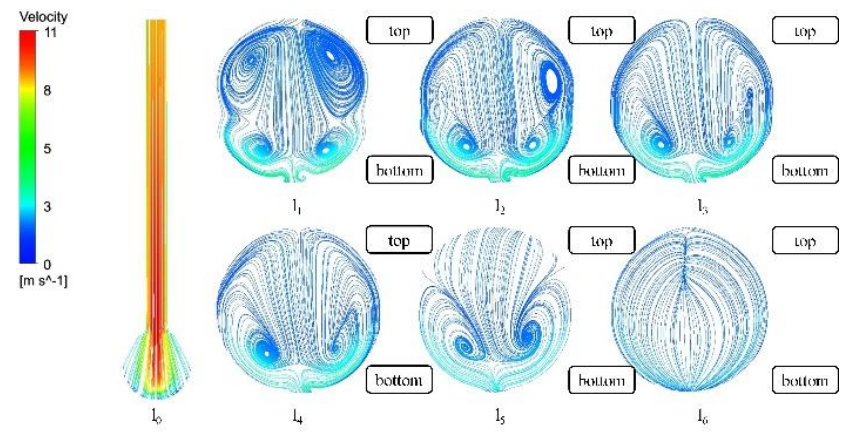

c

Fig. 11 Streamlines in the sections $(\mathrm{a}-d=60 \mathrm{~mm}$; $\mathrm{b}-d=$ $=40 \mathrm{~mm} ; \mathrm{c}-d=20 \mathrm{~mm}, v_{0}=10 \mathrm{~m} / \mathrm{s}, Q_{p}=$ $=0.001 \mathrm{~kg} / \mathrm{s}$ )

As shown in Fig. 11, b, there is no vortex on the sides of outlet pipe when the gas passes through the intersection. At $l_{1}$, two neighbouring coarse vortices appear at the top of the section. At $l_{2}$, two opposite vortices are formed at the top of the section and two neighbouring vortices are formed on the bottom of the section. At $l_{3}$ and $l_{4}$, these two opposite vortices move from the top to the centre of the flow field, while these two neighbouring vortices begin to disappear. At $l_{5}$, these two opposite vortices reach the centre and 
these two neighbouring vortices have disappeared. At $l_{6}$, these two opposite vortices make one vortex.

Fig. 11, c shows no vortex on the sides of outlet pipe when the gas passes through the intersection. At $l_{1}$, two couple opposite vortices are formed in the fluid field. At $l_{2}$ and $l_{3}$, these two upper vortices begin to disappear successively. At $l_{4}$ and $l_{5}$, these two lower vortices become larger and begin to combine. At $l_{6}$, these two couple opposite vortices have disappeared absolutely.

As a conclusion, it is acceptable that the particle mass concentration on the outlet pipe is caused by secondary flow vortices. With the inlet diameter decreases, the gas flow state in the outlet pipe become more complex but lower velocity.

\subsection{Effect of the inlet velocity}

The conditions of $d=20 \mathrm{~mm}, 40 \mathrm{~mm}, 60 \mathrm{~mm}$ are chosen to simulate the effect of inlet velocity. As shown the Fig. 12, with the inlet velocity increases, both the erosion rate on the intersection and the outlet pipe increase. It is observed that the region of erosion occurs only at the intersection of the tee pipe at $v_{0}=10 \mathrm{~m} / \mathrm{s}$. However, four $\mathrm{V}$-shaped erosion scars occur at the sides of the elliptical erosion region next. What's more, the spiral erosion scars appear on the outlet pipe. Generally speaking, the severest erosion region is concentrated at the intersection of the tee pipe.
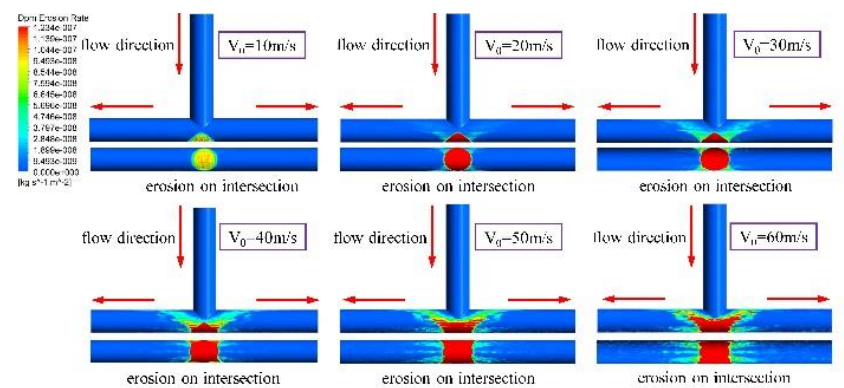

a
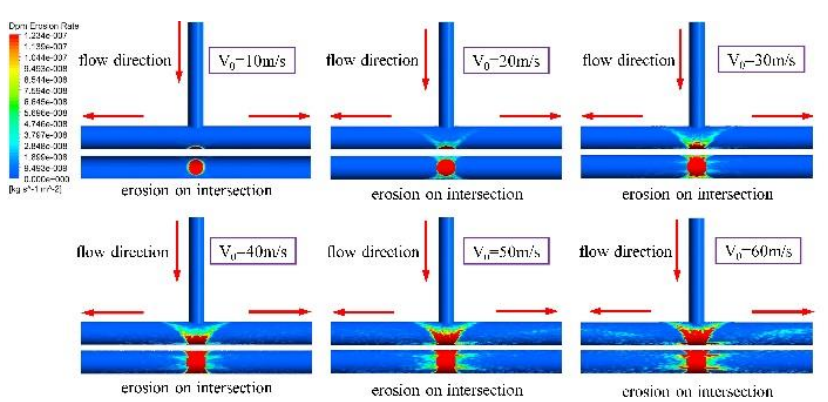

b
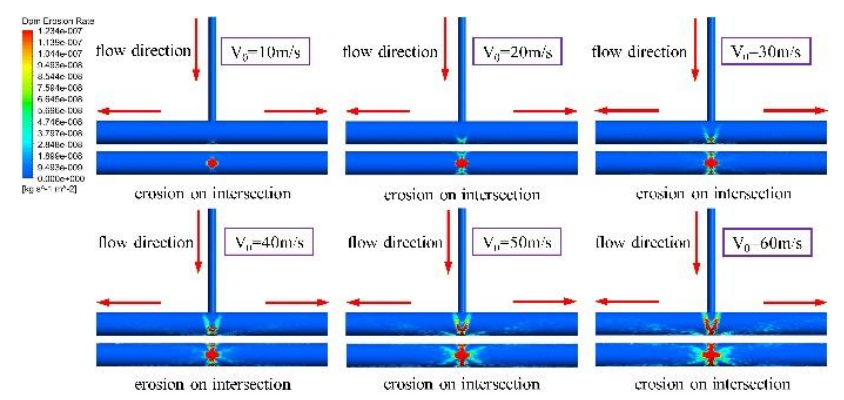

$\mathrm{c}$
It is seen from Fig. 13 that the maximum erosion rate increases with the increasing of inlet velocity. Furthermore, the maximum erosion rate is exponential growth. When the inlet diameter of $20 \mathrm{~mm}$, the region of erosion is small while the erosion rate is higher than other inlet diameter. Therefore, the smaller the inlet diameter, the more erosion rate of the tee pipe. With the inlet velocity increases, the erosion at inlet diameter of $20 \mathrm{~mm}$ is the severest.

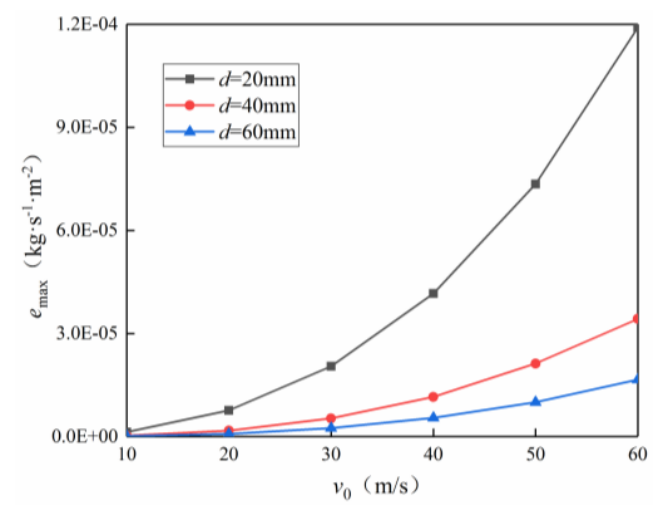

Fig. 13 Inlet velocity and the maximum erosion rate

\subsection{Effect of the particle mass flow rate}

Fig. 14 shows the relationship between the particle mass flow rate and the maximum erosion rate. The larger

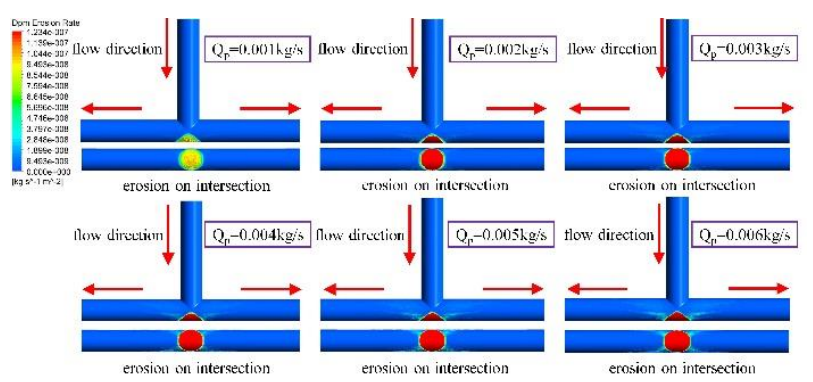

a
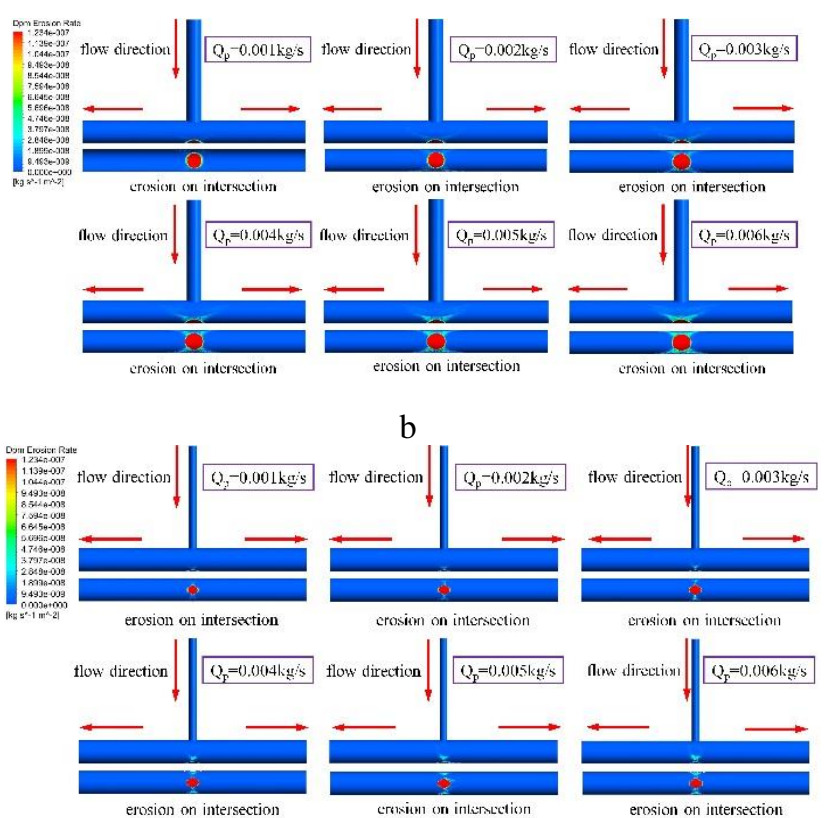

c

Fig. 12 Contours of erosion ( $\mathrm{a}-d=60 \mathrm{~mm} ; \mathrm{b}-d=40 \mathrm{~mm}$; c - $d=20 \mathrm{~mm}, Q_{p}=0.001 \mathrm{~kg} / \mathrm{s}$ )

Fig. 14 Contours of erosion $\mathrm{a}-d=60 \mathrm{~mm} ; \mathrm{b}-d=40 \mathrm{~mm}$; $\mathrm{c}-d=20 \mathrm{~mm}, v_{0}=10 \mathrm{~m} / \mathrm{s}$ 
the particle mass flow rate, the more maximum erosion rate of the tee pipe. The increase of the number of particles in the gas conduces to the increase of the tee pipe erosion. Different from the result in Fig. 12, there are only two V-shaped erosion scars occur at the sides of the intersection. In addition, there are no erosion occur on the outlet pipe.

As shown in Fig. 15, the maximum erosion rate is linear growth. The maximum erosion rate increases quickly when the inlet diameter is $20 \mathrm{~mm}$. However, the growth rate becomes small when the inlet diameters are $40 \mathrm{~mm}$ and 60 $\mathrm{mm}$. Due to the increase of the number of impact particles and the decrease of the impact region, the erosion of the impact region is severer.

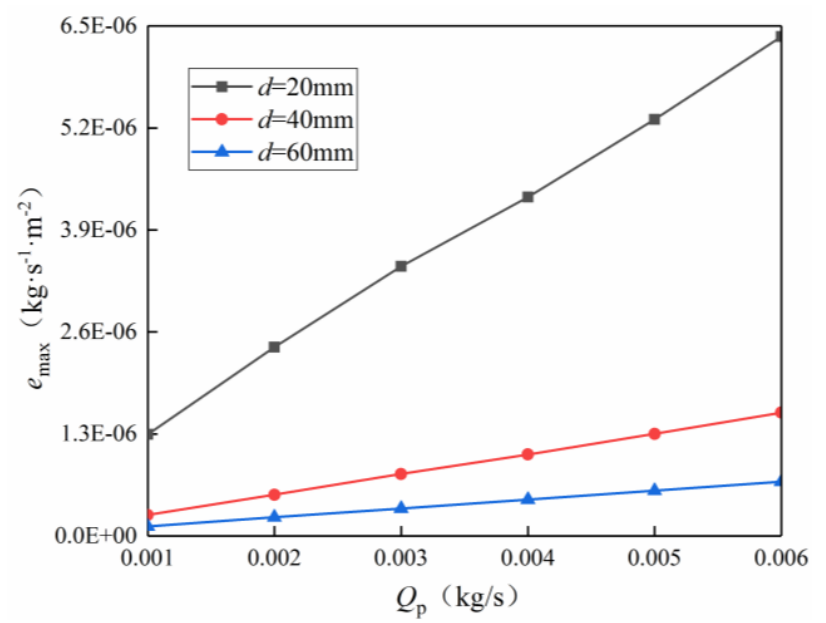

Fig. 15 Particle mass flow rate and the maximum erosion rate

\section{Conclusions}

1. Gas flow direction changes at the intersection of the tee pipe, resulting in the formation of the elliptical highpressure region. This high-pressure region is related to the inlet diameter. The erosion scar is similar to the distribution of particle mass concentration.

2. The larger the inlet diameter is, the lower the turbulence intensity is in the outlet pipe. However, the smaller the inlet diameter, the slower the gas velocity in the outlet pipe. It indicates that the particle mass concentration on the outlet pipe is caused by secondary flow vortices. The emergence and development of these vortices drive the motion of the particles.

3. As a whole, the maximum erosion rate region is concentrated at the intersection of the tee pipe. With the inlet velocity increases, both the erosion rate on the intersection and the outlet pipe increase. What's more, four V-shaped erosion scars occur at the sides of the elliptical erosion region next. However, there are only two V-shaped erosion scars occur at the sides of the intersection. The larger the particle mass flow rate, the more maximum erosion rate of the tee pipe.

\section{Acknowledgment}

This work is supported by Open Fund of National Joint Engineering Research Centre for Abrasion Control and Moulding of Metal Materials (HKDNM201806), and Sichuan Science and Technology Project (2020JDRC0088).

\section{References}

1. Mazdak, P.; Kamyar, N.; Fardis, N.; Shokrollah, H.; Brenton, S. M.; Siamack, A. S. 2014. A comprehensive review of solid particle erosion modeling for oil and gas wells and pipelines applications, Journal of Natural Gas ence \& Engineering 21: 850-873. http://dx.doi.org/10.1016/j.jngse.2014.10.001.

2. Fan, C.; Xu, J.; Yang, T.; Fu, H. L. 2014. Research on a measurement method for wheat hardness based on the erosion effects of laser ultrasonics, Laser Physics 24(4): 1211-1214. http://dx.doi.org/10.1088/1054-660X/24/4/046101.

3. Xu, L.; Zhang, Q.; Zheng, J.; Zhao, Y. 2016. Numerical prediction of erosion in elbow based on CFD-DEM simulation, Powder Technology 302: 236-246. http://dx.doi.org/10.1016/j.powtec.2016.08.050.

4. Karimi, S.; Shirazi, S.; Melaury, B. 2016. Predicting fine particle erosion utilizing computational fluid dynamics, Wear 376-377: 1130-1137. http://dx.doi.org/10. 1016/j.wear.2016.11.022.

5. Zeng, D.; Zhang, E.; Ding, Y.; Yi, Y.; Xian, Q.; Yao, G.; Hong, Z.; Tai, S. 2018. Investigation of erosion behaviors of sulfur-particle-laden gas flow in an elbow via a CFD-DEM coupling method, Powder Technology 329: $115-128$. https://doi.org/10. 1016/j.powtec.2018.01.056.

6. Xu, L.; Sun, Q.; Tang, B.; Huang, C. X.; Li, J. 2018. Numerical simulation of ice particle erosion in seawater pipelines of polar ship under vibration conditions, Ocean Engineering 147(1): 9-19. http://dx.doi.org/10.1016/j.oceaneng.2017.10.019.

7. Wang, K.; Li, X.; Wang, Y.; He, R. 2017. Numerical investigation of the erosion behavior in elbows of petroleum pipelines, Powder Technology 314: 490-499. http://dx.doi.org/10.1016/j.powtec.2016.12.083.

8. Qiao, Q.; Cheng, G.; Li, Y.; Wu, W.; Hu, H. 2017. Corrosion failure analyses of an elbow and an elbow-topipe weld in a natural gas gathering pipeline, Engineering Failure Analysis 82: 599-616. http://dx.doi.org/10.1016/j.engfailanal.2017.04.016.

9. Pei, J.; Lui, A.; Zhang, Q.; Xiong, T.; Jiang, P.; Wei, W. 2018. Numerical investigation of the maximum erosion zone in elbows for liquid-particle flow, Powder Technology 333: 47-59. http://dx.doi.org/10.1016/j.powtec.2018.04.001.

10. Zhou, J. W.; Liu, Y.; Liu, S. Y.; Du, C.; Li, J. 2017. Effects of particle shape and swirling intensity on elbow erosion in dilute-phase pneumatic conveying, Wear 380381: 66-77. http://dx.doi.org/10. 1016/j.wear.2017.03.009.

11. Zhang Jixin; Bai Yongqiang; Kang Jian Wu Xuan. 2017. Failure analysis and erosion prediction of tee junction in fracturing operation, Journal of Loss Prevention in the Process Industries 46: 94-107. http://dx.doi.org/10.1016/j.jlp.2017.01.023.

12. Banakermani, M. R.; Naderan, H.; Saffar-Avval, M. 2018. An investigation of erosion prediction for $15 \mathrm{de}-$ grees to 90 degrees elbows by numerical simulation of gas-solid flow, Powder Technology 334: 9-26. http://dx.doi.org/10.1016/j.powtec.2018.04.033. 
13. Arsalanloo, A.; Abbasalizadeh, M. 2017. Numerical study on deposition of particles in a $90^{\circ}$ bend in the presence of swirling flow using Eulerian-Lagrangian method, Powder Technology 320: 285-294. http://dx.doi.org/10.1016/j.powtec.2017.07.050.

14. Zhu, H. J.; Li, S. 2018. Numerical analysis of mitigating elbow erosion with a rib, Powder Technology 330: 445-460. http://dx.doi.org/10.1016/j.powtec.2018.02.046.

15. Duarte, C. A. R.; de Souza, F. J. 2017. Innovative pipe wall design to mitigate elbow erosion: A CFD analysis, Wear 381: 176-190. http://dx.doi.org/10.1016/j.wear.2017.03.01.5.

16. Parsi, M.; Al-Sarkhi, A.; Kara, M.; Sharma, P.; Mclaury, B. S.; Shirazi, S. A. 2017. A new dimensionless number for solid particle erosion in natural gas elbows, Wear 390-391: 80-83. http://dx.doi.org/10.1016/j.wear.2017.07.005.

17. Parsi, M.; Kara, M.; Agrawal, M.; Kesana, N.; Jatale, A.; Sharma, P.; Shirazi, S. 2016. CFD simulation of sand particle erosion under multiphase flow conditions, Wear 376: 1176-1184.

http://dx.doi.org/10.1016/j.wear.2016.12.021.

18. Zamani, M.; Seddighi, S.; Nazif, H. R. 2017. Erosion of natural gas elbows due to rotating particles in turbulent gas-solid flow, Journal of Natural Gas Science and Engineering 40: 91-113.

http://dx.doi.org/10.1016/j.jngse.2017.01.034.

19. Wang, J.; Shirazi, S. A. 2003. A CFD Based Correlation for Erosion Factor for Long-Radius Elbows and Bends, Journal of Energy Resources Technology 125(1): 26-34. http://dx.doi.org/10.1115/1.1514674.

20. Varga, M.; Goniva, C.; Adam, K.; Badisch, E. 2013. Combined experimental and numerical approach for wear prediction in feed pipes, Tribology International 65: 200-206. http://dx.doi.org/10.1016/j.triboint.2013.02.014.

21. El-Behery, S. M.; Hamed, M. H.; Ibrahim, K. A.; ElKadi, M. A. 2010. CFD Evaluation of Solid Particles Erosion in Curved Ducts, Journal of Fluids Engineering 132(7): 1-10. http://dx.doi.org/10.1115/1.4001968.

22. Jie Zhang, Yang Hu. 2020. Sealing performance and mechanical behavior of PEMFCs sealing system based on thermodynamic coupling, International Journal of Hydrogen Energy 45(43): 23480

https://doi.org/10.1016/j.ijhydene.2020.06.167.
23. Oka, Y. I.; Okamura, K.; Yoshida, T. 2005. Practical estimation of erosion damage caused by solid particle impact: Part 1: Effects of impact parameters on a predictive equation, Wear 259(1-6): 95-101. http://dx.doi.org/10.1016/j.wear.2005.01.039.

24. Jie Zhang, Jingxuan Xie. 2020. Effect of reservoir's permeability and porosity on the performance of cellular development model for enhanced geothermal system, Renewable Energy 148: 824-838. http://dx.doi.org/10.1016/j.renene.2019.10.168.

25. Grant, G.; Tabakoff, W. 2012. Erosion Prediction in Turbomachinery Resulting from Environmental Solid Particles, Journal of Aircraft 12(5): 471-478. http://dx.doi.org/10.2514/3.59826.

\section{J. Hu, H. Zhang, J. Zhang, S. Niu, W. Cai \\ GAS-SOLID EROSION WEAR CHARACTERISTICS OF TWO-PHASE FLOW TEE PIPE}

\section{S u m m a r y}

Particles erosion wear always consist in the intersection of tee pipe, which is an inevitable problem. In order to obtain the erosion wear characteristics of two-phase flow tee pipe, several cases of different inlet diameters are investigated numerically in this paper. Euler-Lagrange method is adopted to describe the gas-solid two-phase flow and the finite volume method is adopted to solve the erosion results. Meshing O-type grids to obtain the reasonable boundary layer in ICEM CFD. By verifying and comparing the turbulence intensity and velocity of the six meshes, a reasonable finite element model is selected. Intersection, the severest erosion region, is the location where the gas flow direction changes. The inlet diameter determines the region of the impact particles directly hitting the wall. When the inlet diameter is smaller, the erosion rate of the intersection become more. As the inlet velocity increases, both the erosion rate and erosion scars of the intersection and the outlet pipe become more. However, there are only the erosion scars at the intersection are affected, with the increase of particle mass flow.

Keywords: two-phase flow, tee pipe, erosion, inlet diameter.

Received August 11, 2020

Accepted June 02, 2021 